\title{
Avaliação da qualidade de cuidados de enfermagem em hospital público
}

\section{Quality evaluation of nursing care in public hospital}

\author{
Graziela Caldana ${ }^{1}$; Carmen Silvia Gabriel²; Andrea Bernardes ${ }^{3}$; Rosicler Xelegati \\ de Pádua ${ }^{4}$; Dagmar Willamowius Vituri ${ }^{5}$; Mariana Angela Rossaneis ${ }^{6}$
}

\section{Resumo}

O estudo objetivou avaliar a qualidade dos cuidados de enfermagem em um hospital público de um município do interior do Estado de São Paulo a partir de indicadores de estrutura, processo e resultados, por meio da observação direta de pacientes e da análise de prontuários utilizando-se um instrumento de registro de busca ativa. Foram realizadas 720 observações e utilizada a estatística descritiva para verificar o percentual de adequação dos cuidados observados em relação ao padrão de qualidade proposto para os indicadores. Dos 12 indicadores avaliados, dois apresentaram-se dentro do índice de conformidade ideal: "pacientes com infusão venosa sem lesões cutâneas pós-infiltrativas, associadas à punção venosa para infusão de soro e/ou medicações/dia" e "pacientes com sonda nasoenteral recebendo dieta com cabeceira elevada a $30^{\circ}$ ". Os demais receberam um índice de conformidade inferior ao preconizado, mas nenhum abaixo $75 \%$, demonstrando um compromisso da equipe de enfermagem com a qualidade da assistência prestada.

Descritores: Garantia da Qualidade dos Cuidados de Saúde. Indicadores de Qualidade em Assistência à Saúde. Serviço Hospitalar de Enfermagem.

\begin{abstract}
The study aimed to evaluate the quality of nursing care in a public hospital, of a municipality in the São Paulo State, based on indicators of structure, process and results, through direct observation of patients and patient records using an instrument of active search record". 720 observations were made and was used the descriptive statistics to verify the percentage of adequacy of care observed to the pattern of quality care proposed on the indicators. From the 12 indicators assessed, two were within the conformity index ideal: "Patients with venous infusion without post-infiltrative skin lesions, associated
\end{abstract}

\footnotetext{
${ }^{1}$ Enfermeira, discente do Programa de Doutorado em Enfermagem Fundamental pela Escola de Enfermagem de Ribeirão Preto Universidade de São Paulo. E-mail: graziela.caldana@usp.br (contato principal para correspondência).

${ }^{2}$ Enfermeira, Prof ${ }^{\mathrm{a}}$. Dr ${ }^{\mathrm{a}}$. do Departamento de Enfermagem Geral e Especializada da Escola de Enfermagem de Ribeirão Preto USP , Brasil. E-mail: cgabriel@eerp.usp.br.

${ }^{3}$ Enfermeira, Profa ${ }^{\mathrm{a}}$. Dr ${ }^{\mathrm{a}}$. do Departamento de Enfermagem Geral e Especializada da Escola de Enfermagem de Ribeirão Preto USP , Brasil. E-mail: andreab@eerp.usp.br.

${ }^{4}$ Enfermeira, Mestre em Ciências, Especialista em Laboratório do Departamento de Enfermagem Geral e Especializada da Escola de Enfermagem de Ribeirão Preto USP, Brasil. E-mail: rxelegati@eerp.usp.br.

${ }^{5}$ Mestre em Enfermagem. Enfermeira da Assessoria de Controle da Qualidade da Assistência de Enfermagem do Hospital Universitário Regional do Norte do Paraná. Universidade Estadual de Londrina, Londrina, Brasil. E-mail: dagmar@uel.br.

${ }^{6}$ Enfermeira. Mestranda no Programa de Pós-Graduação em Enfermagem Fundamental da Escola de Enfermagem de Ribeirão Preto - USP. Universidade Estadual de Londrina. E-mail: marianarossaneis@gmail.com.
} 
with venipuncture for infusion of serum and/or medications/day" and "Patients with nasogastric tube receiving diet with high headboard at 30 degrees". The others received a compliance index lower than recommended, but none below $75 \%$, demonstrating a nursing team's commitment with quality of assistance provided.

Descriptors: Quality Assurance. Quality Indicators. Nursing Service Hospital.

\section{Introdução}

A qualidade não pode ser definida de maneira universal e abrangente para todos os segmentos por depender de fatores individuais, culturais, religiosos e ambientais. O setor saúde foi um dos últimos a aderir ao movimento de busca pela qualidade, mesmo nos países como Canadá e Estados Unidos, o que se deve a disputas acirradas de mercado no setor (FELDMAN; CUNHA, 2006).

Com relação ao Brasil, a abordagem da gestão pela qualidade na saúde ainda é incipiente devido à falta de competitividade entre as instituições de saúde e uma tímida participação social no que tange aos direitos da população em relação aos serviços de saúde.

Nesse contexto, qualidade consiste em obter os maiores benefícios por meio de menores riscos e, portanto, para sua avaliação, estabelece-se uma tríade que consiste na estrutura, no processo e no resultado. A estrutura pode ser entendida como recursos físicos, humanos, materiais, financeiros e equipamentos necessários para a assistência à saúde; o processo refere-se às atividades envolvendo profissionais de saúde e usuários, incluindo diagnóstico, tratamento, aspectos éticos de relação profissional; o resultado corresponde ao produto final da assistência prestada, considerando a saúde, a satisfação de padrões e as expectativas dos usuários (DONABEDIAN, 1999).

A busca da qualidade consiste numa questão complexa, devendo ser prioridade para as instituições de saúde e para os profissionais que as integram. Dentre estes, destacam-se os profissionais de enfermagem, os quais possuem um papel fundamental nas organizações de saúde, tendo como foco a assistência individualizada e adequada às melhores práticas de qualidade e segurança.
Atualmente, as instituições de saúde em todo o mundo são estimuladas a prestar um cuidado seguro e de alta qualidade ao paciente, apesar da limitação de equipamentos ou recursos financeiros e humanos. Contudo, a maioria dessas instituições tem dificuldade em reconhecer o que realmente deve ser implementado para fornecer segurança ao paciente e qualidade aos serviços prestados. Muitas instituições tem insegurança, por exemplo, como começar a estruturar seus esforços de redução de riscos. Considera-se fundamental que as organizações saibam quais intervenções terão o maior impacto na qualidade e na segurança dos pacientes (JOINT COMMISSION INTERNATIONAL, 2010).

Para que se possa desenvolver uma cultura de avaliação da qualidade, é necessário atuar com base em parâmetros alcançáveis e adequados a cada realidade e a mensuração destes parâmetros pode se dar na forma de indicadores, os quais são representações, quantitativas ou não, de resultados, ocorrências ou eventos de um sistema, constituindose um instrumento gerencial de mensuração sem o qual é impossível a avaliação de critérios (BITTAR, 1996).

Resultados de estudo de levantamento da produção científica da enfermagem sobre a utilização de indicadores de desempenho nos serviços de enfermagem hospitalar apontam que, em um total de 74 publicações, apenas 15 destacavam a importância do uso dos indicadores de estrutura, processo ou resultado. Tal fato demonstra que ainda há pouco conhecimento disponível acerca da temática e que estudos que avaliem os resultados do cuidado de enfermagem são necessários e fundamentais para subsidiar novas práticas e pesquisas voltadas à qualidade (CALDANA et al., 2011; VITURI, 2007). 
A qualidade do cuidado de enfermagem geralmente é avaliada sob a ótica da estrutura e do processo, pois possibilitam dados mais objetivos e concretos. Estudiosos corroboram com essa afirmação, quando assumem que os indicadores mais estudados são aqueles que se relacionam aos cuidados físicos, ou seja, de processo (IDVALL et al., 1997; TRNOBRANSKI, 1993). Segundo a mesma revisão de literatura, 74\% dos indicadores utilizados para avaliação de serviços de enfermagem são de processo, $16,6 \%$ são de estrutura e $9,4 \%$ são indicadores de resultado. Observa-se que o foco dos indicadores identificados está fortemente apontado para os processos assistenciais da enfermagem.

Estudos que apontam e utilizam indicadores de desempenho voltados à avaliação do resultado do cuidado sãonecessários e fundamentais para subsidiar novas práticas e pesquisas. Assim, questiona-se: qual é a qualidade da assistência de enfermagem de um hospital público de acordo com indicadores de desempenho pré-estabelecidos? Tendo em vista a necessidade de se investir em sistemas de avaliação da qualidade do cuidado de enfermagem, este estudo teve como objetivo avaliar a prática dos cuidados de enfermagem em um hospital público do interior do Estado de São Paulo, tendo por base, indicadores de estrutura, processo e resultados.

\section{Material e Método}

Trata-se de um estudo do tipo exploratório, com abordagem quantitativa. O local escolhido foi um hospital localizado em um município do interior do Estado de São Paulo, entidade pública, estadual com 50 leitos, inaugurado em 2008 com o objetivo de atender aos municípios do Departamento Regional de Saúde no que tange a atenção hospitalar de média complexidade. A instituição está subordinada à Secretaria de Estado da Saúde do Estado de São Paulo (SESSP), prestando serviços no modelo de contratualização com o Sistema Único de Saúde (SUS). A administração é intermediada por uma fundação privada, seguindo o Modelo de Organização Social de Saúde que vem sendo adotado pelo Governo Estadual desde o final da década de 90. Divide-se em seis unidades e o organograma é composto por quatro diretores médicos e uma coordenadora de enfermagem. No quadro da enfermagem, há 27 enfermeiros, todos subordinados à coordenadora de enfermagem, sem cargos intermediários de chefia e distribuídos linearmente pelas seis unidades da instituição (HOSPITAL ESTADUAL DE RIBEIRÃO PRETO, 2011).

O hospital integra o Programa "Compromisso com a Qualidade Hospitalar" (CQH), que tem como missão contribuir para a melhoria contínua da qualidade do atendimento nos serviços de saúde e procura incentivar a utilização dos indicadores hospitalares como suporte ao processo decisório. Também foi eleito como o Melhor Hospital Público do Estado de São Paulo, tendo ficado em primeiro lugar na Pesquisa de Satisfação dos Usuários do Sistema Único de Saúde em 2010 (HOSPITAL ESTADUAL DE RIBEIRÃO PRETO, 2011).

A amostra desta pesquisa foi composta por pacientes atendidos na unidade de clínica médica. Para a seleção da amostra, foram utilizados os seguintes critérios: internação na clinica médica, cadastro na instituição, idade superior a dezoito anos, concordância na participação da pesquisa e assinatura do Termo de Consentimento Livre e Esclarecido.

Foram realizadas de 28 a 30 observações/dia, no período de janeiro a março de 2011, totalizando 25 dias de coleta por avaliadores capacitados pelo pesquisador principal a utilizar o instrumento com vistas a padronizar as observações. Ao final da coleta, totalizaram-se 720 observações.

A coleta de dados ocorreu por meio de observação direta do paciente e de seu prontuário, os itens observados estavam descritos num instrumento de registro de busca ativa, desenvolvido por Vituri (2007). Ressalta-se que, após a autorização da autora do instrumento foram realizadas as seguintes 
adaptações: inclusão das escalas de avaliação de risco para úlcera por pressão (BERGSTROM; BRADEN; LAGUZZA, 1987) e risco para queda (MORSE, 1997), no intuito de conferir maior fidedignidade às observações. Os itens observados foram os seguintes: leitos com identificação adequada; pulseiras com identificação adequada; pacientes com risco de queda do leito com grade lateral/dia; acessos venosos periféricos com identificação adequada/dia; pacientes com infusão venosa sem lesões cutâneas pós- infiltrativas, associadas à punção venosa para infusão de soro e/ ou medicações/dia; equipos macro gotas, equipos de bomba infusora, nutrição parenteral ou equipos de PVC com identificação adequada/dia; rótulos de soro com identificação adequada; pacientes com sonda nasoenteral recebendo dieta com cabeceira elevada a $30^{\circ}$ ou mais; sondas vesicais de demora com fixação adequada; pacientes com risco ou com UPP com colchão adequado para prevenção; rotina de sinais vitais verificada de forma completa; prescrição checada de forma completa.

A análise dos prontuários, em suporte de papel e em registro eletrônico, foi realizada com o intuito de observar a prescrição médica e registro de verificação de sinais vitais.

Como guia para preenchimento do instrumento e para posterior análise do grau de conformidade de cada indicador observado, foi utilizado um manual operacional também adaptado do estudo de Vituri (2007) no qual contempla, para cada um dos indicadores propostos, um descritor, o referencial científico que fundamentou o padrão determinado no descritor, o numerador e denominador, bem como o cálculo do mesmo, a fonte dos dados, a amostra para análise de conformidade, a periodicidade das avaliações e os itens de avaliação do indicador que constam no instrumento proposto.

Além destas informações, consta no manual o Índice de Conformidade Ideal (ICI), ou seja, o percentual de adequação esperado em relação ao padrão determinado - scoring da medida. Este preconiza que deve haver $100 \%$ de conformidade para todos os indicadores descritos, exceto para o indicador " $\mathrm{N}^{\mathrm{o}}$ de pacientes com infusão venosa sem lesões cutâneas pós-infiltrativas, associadas à punção venosa para infusão de soro e/ou medicações/ dia", que possui um índice de conformidade ideal de $80 \%$, pois as lesões cutâneas pós infiltrativas podem não ser exclusivamente associadas à técnica de punção, podendo estar relacionadas às condições inerentes ao próprio paciente, às condições da veia, ao tipo e pH da medicação ou solução em uso, calibre, tamanho, comprimento e material do cateter utilizado na punção que, poderiam influenciar na condição avaliada pelo indicador dezessete (PHILLIPS, 2001; FERREIRA, 2005).

Cabe destacar que a observação se deu após a apresentação dos objetivos e dos aspectos éticos ao sujeito participante e/ou responsável e que a coleta de dados apenas se realizou após a aprovação do Comitê de Ética em Pesquisa com Seres Humanos da Escola de Enfermagem de Ribeirão Preto da Universidade de São Paulo (Protocolo CEP/EERP número 1192/2010).

\section{Resultados e Discussão}

Dos 12 indicadores avaliados, dois $(18,1 \%)$ apresentaram-se dentro do índice de conformidade determinado no manual operacional, a saber: "pacientes com infusão venosa sem lesões cutâneas pós-infiltrativas, associadas à punção venosa para infusão de soro e/ou medicações/dia" e "pacientes com sonda nasoenteral recebendo dieta com cabeceira elevada a $30^{\circ}$ ou mais".

Dentre os dez itens que apresentaram não conformidade de acordo com o manual operacional, observamos na tabela 1 , que o percentual em relação à "pacientes identificados com pulseira" (76,3\%) e "pacientes com risco ou com úlcera por pressão (UPP) com colchão adequado para prevenção" (77\%) foram os indicadores que apresentaram o maior desvio em 
relação ao padrão, acima de $20 \%$. Dois indicadores que apresentaram variação menor que $3 \%$ em relação ao preconizado foram: "rótulos de soro com identificação adequada" (98,8\%) e "rotina de sinais vitais verificada de forma completa" $(98,2 \%)$.

Tabela 1 - Relação dos indicadores de qualidade e o índice de conformidade alcançado.

\section{Indicadores de qualidade}

Leitos com identificação adequada

Pulseiras com identificação adequada

Pacientes com risco de queda do leito com grade lateral/dia

Acessos venosos periféricos com identificação adequada/dia

Pacientes com infusão venosa sem lesões cutâneas pós- infiltrativas, associadas à punção venosa para infusão de soro e/ou medicações/dia.

Equipos macro gotas, equipos de bomba infusora, nutrição parenteral ou equipos de PVC com identificação adequada/dia.

Rótulos de soro com identificação adequada

Pacientes com sonda nasoenteral recebendo dieta com cabeceira elevada a $30^{\circ}$ ou mais

Sondas vesicais de demora com fixação adequada.

Pacientes com risco ou com UPP com colchão adequado para prevenção

Rotina de Sinais vitais verificada de forma completa

Prescrição checada de forma completa
Índice de conformidade

$88,3 \%$

$76,3 \%$

$90,4 \%$

$79,2 \%$

$98,0 \%$

$87,7 \%$

$98,8 \%$

$100,0 \%$

$95,6 \%$

$77,0 \%$

$98,2 \%$

$97,8 \%$

Fonte: Hospital Estadual de Ribeirão Preto (2011).

Os resultados demonstram que nenhum dos indicadores avaliados obteve uma conformidade menor que $75 \%$, o que pode ser relacionado a um compromisso dos trabalhadores de enfermagem da instituição em atender bons padrões de qualidade em seus processos assistenciais.

Dos indicadores avaliados que obtiveram destaque em relação aos demais por alcançarem o ICI determinado pelo manual operacional apontam a preocupação e responsabilidade da equipe de enfermagem em prestar um cuidado com qualidade.

Devido aos quadros clínicos de alta complexidade e que muitas vezes representam risco de vida, os pacientes tem sido submetidos cada vez mais a procedimentos invasivos que demandam conhecimento por parte dos profissionais que os executam. Em estudo realizado no Rio de Janeiro, o autor afirma que a prescrição, o planejamento e a administração da terapia, a escolha dos tipos de cateteres e acessórios de infusão, a obtenção do acesso, o preparo e as técnicas de administração de fármacos e soluções, os cuidados na manutenção do acesso, o controle das infusões, a prevenção de complicações e a monitorização constante estão entre as principais ações para a promoção da eficácia e segurança da criança em terapia intravenosa (CARDOSO et al., 2011). 
Quanto ao indicador relacionado à utilização de sonda nasoenteral e a elevação da cabeceira, estudo aponta que a elevação do decúbito previne acidentes que decorrem de regurgitação e vômitos, que podem ter como consequências a aspiração pulmonar principalmente em pacientes inconsciente, neurológico ou idoso. Sendo assim, esse deve ser em hábito dos profissionais de enfermagem, pois é essencial para a segurança do paciente (HERMANN; CRUZ, 2008).

Em relação aos indicadores que abordam a identificação do paciente, o indicador "Leito com identificação adequada" atingiu maior conformidade (88,3\%); em relação ao indicador "Pulseira com identificação adequada", que atingiu 76,3\%. Ambos indicadores são medidas essenciais de segurança do paciente; por meio deles, os profissionais de saúde podem assegurar a correta identificação do paciente e, assim, realizar os procedimentos pertinentes ao seu tratamento clínico (MIASSO; CASSIANI, 2000).

Estudo realizado no interior do estado de São Paulo ressalta que os indicadores são ferramentas fundamentais para avaliação da qualidade por apontarem aspectos do cuidado que podem ser melhorados, tornando a assistência aos pacientes livre de riscos e falhas e, portanto, mais segura (NASCIMENTO, 2008).

Um dos indicadores avaliados também foi "rotina de sinais vitais verificada de forma completa", com 98,2\% de adequação. O indicador "prescrição checada de forma completa" também se destacou quanto á sua adequação, com 97,8\%, o que demonstra o comprometimento da equipe de enfermagem com a realização das atividades que lhes são atribuídas, pois a checagem indica que tais atividades foram realizadas.

Estudo realizado em um hospital universitário de grande porte da cidade de São Paulo aponta que, em $70,7 \%$ dos prontuários, apenas $1 \%$ estava completo e $24,8 \%$ do item checagem das prescrições de enfermagem não foi aplicado porque as prescrições estavam ausentes (SETZ; D'INNOCENZO, 2009). Estes resultados evidenciam o destaque da instituição em estudo quanto a este indicador quando comparado com o artigo citado anteriormente.

Quanto ao indicador "sondas vesicais de demora com fixação adequada", o percentual atingido pela equipe de enfermagem foi de 95,6\%. O cuidado relacionado a este indicador é muito importante, pois a fixação inadequada ou a ausência de fixação da sonda vesical de demora expõem o paciente ao risco de lesões traumáticas na uretra resultantes da tração da sonda (POTTER; PERRY, 2009).

O indicador "rótulos de soro com identificação adequada" atingiu percentual de adequação de 98,8\%, estando, assim, dentro do padrão estabelecido pelo manual operacional. Essa identificação é importante para que erros com administração de medicamentos sejam evitados.

Em relação ao indicador "número de acessos venosos periféricos com identificação adequada/ dia", este obteve $79,2 \%$ de adequação. O indicador "equipos macrogotas, equipos de bomba infusora, nutrição parenteral ou equipos de PVC com identificação adequada/dia" atingiu 87,7\% de adequação. Tais porcentagens sinalizam que mais atenção deve ser dada a identificação correta dos itens relacionados a estes indicadores, pois a sua utilização, segundo a Agência Nacional de Vigilância Epidemiológica - ANVISA, está amplamente associada à infecção primária de corrente sanguínea. A ANVISA ainda recomenda que os profissionais da saúde estejam atentos à troca do sistema de infusão no tempo adequado (BRASIL, 2012).

Neste estudo, utilizar indicadores como ferramenta de avaliação da qualidade da assistência proporcionou mensurar e comparar os valores alcançados com o ICI. Nota-se que a utilização de indicadores pela enfermagem ajuda a prevenir possíveis eventos adversos, como a ocorrência de queda do paciente, úlcera por pressão, erros na 
identificação e administração de medicamentos, os quais comprometem a qualidade do cuidado e a segurança do paciente.

\section{Conclusão}

O estudo permitiu avaliar a qualidade da assistência de enfermagem de um hospital público à luz de indicadores pré-estabelecidos apontando que, dos 12 indicadores de qualidade avaliados 16,6\% apresentaram conformidade ideal, dos que não tiveram conformidade ideal, 66,6\% apresentavam um desvio de conformidade menor que 20\%, o que permite concluir que, tomando por base os indicadores avaliados há um compromisso do serviço de enfermagem, com bons padrões de qualidade. A avaliação da qualidade da assistência tendo em vista indicadores padrão deveria se tornar cada vez mais presentes nos Serviços de Enfermagem hospitalar, os quais precisam se comprometer com o pleno atendimento das necessidades de seus clientes internos e externos, buscando aumentar o nível de satisfação dos mesmos.

O levantamento dos indicadores avaliados nesta pesquisa trará aos enfermeiros parâmetros para identificar e avaliar a qualidade da assistência prestada. Com base nos resultados obtidos, poderão ser estabelecidas metas e propostas de intervenções que possibilitem a melhoria e gerência do cuidado.

A adaptação do instrumento utilizado, com a inclusão das escalas de avaliação de risco para úlcera por pressão e para queda, tornou os resultados mais fidedignos em relação à identificação dos pacientes que apresentaram risco para tais eventos.

A lacuna encontrada nessa pesquisa refere-se à limitação do estudo em apenas uma instituição, tendo em vista que o sistema de saúde brasileiro conta com uma grande parcela de serviços suplementares, pretende-se reproduzir a pesquisa em um hospital privado a fim de comparar com os resultados deste estudo.

A avaliação da qualidade do cuidado de enfermagem por meio de indicadores deve ser utilizada como instrumento para os profissionais da saúde visando melhoria da assistência prestada, ao mesmo tempo em que consiste numa das formas de compreender a qualidade desse cuidado.

\section{Referências}

BERGSTROM, N.; BRADEN, B.; LAGUZZA, A. The Braden scale for predicting pressure sore risk. Nursing Research, New York, v. 36, n. 4, p. 205210, 1987.

BITTAR, O. J. N. V. Produtividade em hospitais de acordo com alguns indicadores hospitalares. Revista de Saúde Pública, São Paulo, v. 30, n. 1, p. 53-60, 1996.

BRASIL. Ministério da Saúde. Agência Nacional de Vigilância Sanitária (ANVISA). Orientações para prevenção de infecção primária de corrente sanguínea. Unidade de Investigação e Prevenção das Infecções e dos Efeitos Adversos - UIPEA, 2010. p. 1-53. Disponível em: $<$ http://portal.anvisa.gov.br/wps/wcm/connect/ ef02c3004a04c83ca0fda9aa19e2217c/manual+Fin al+preven $\% \mathrm{C} 3 \% \mathrm{~A} 7 \% \mathrm{C} 3 \% \mathrm{~A} 3 \mathrm{o}+\mathrm{de}+\mathrm{infec} \% \mathrm{C} 3 \% \mathrm{~A}$ $7 \% \mathrm{C} 3 \% \mathrm{~A} 3 \mathrm{o}+\mathrm{da}+$ corrente.pdf?MOD=AJPERES.> Acesso em: 26 fev. 2012.

CALDANA, G.; GABRIEL, C. S.; BERNARDES, A.; ÈVORA, Y. D. M. Indicadores de desempenho em serviço de enfermagem hospitalar: revisão integrativa. Revista da Rede de Enfermagem do Nordeste, Fortaleza, v. 12, n. 1, p. 189-97, 2011.

CARdoso, J. M. R. M.; RODRIGUES, E. C.; RODRIGUES, B. M. R. D.; PACHECO, S. T. A.; FARIA, J. C. O. Escolha de veias periféricas para terapia intravenosa em recém-nascidos pela equipe de enfermagem. Revista da Rede de Enfermagem do Nordeste, Fortaleza, v. 12, n. 2, p. 365-73, 2011.

DONABEDIAN, A. The definition of quality and approaches to its assessment. Ann Arbor: Health administration Press; 1999. 
FELDMAN, L. B.; CUNHA, I. C. K. O. Identificação dos critérios de avaliação de resultados do serviço de enfermagem nos programas de acreditação hospitalar. Revista Latino-Americana de Enfermagem, Ribeirão Preto, v. 14, n. 4, p. 540-545, 2006.

FERREIRA, N. M. L. A.; MARASSI, R. P. Avaliando condutas na preservação da infusão venosa no doente hospitalizado. Prática Hospitalar, São Paulo, v. 7, n. 39, 2005. Disponível em: <http:// www.praticahospitalar.com.br/pratica\%2039/pgs/ materia\%2012-39.html>. Acesso em: 26 jan. 2012.

HERMANN, A. P.; CRUZ, E. D. A. Enfermagem em nutrição enteral: investigação do conhecimento e da prática assistencial em hospital de ensino. Cogitare Enfermagem, v. 13, n. 4, p. 520-525, 2008.

HOSPITAL ESTADUAL DE RIBEIRÃO PRETO HERP. Relatório de atividades. Ribeirão Preto, 2011.

IDVALL, E.; ROOKE, L.; HAMRIN, E. Quality indicators in clinical nursing: a review of the literature. Journal of Advanced Nursing, Oxford, v. 25, n. 1, p. 6-17, 1997.

JOINT COMMISSION INTERNATIONAL JCI. The international essentials of health care quality and patient safety. 2010. Disponível em: $<\mathrm{http}$ ://pt.jointcommissioninternational.org/enpt/ International-Essentials-for-Quality-and-PatientSafety>. Acesso em: 25 fev. 2012.

MIASSO, A. I.; CASSIANI, S. H. B. Erros na administração de medicamentos: divulgação de conhecimentos e identificação do paciente como aspectos relevantes. Revista da Escola de Enfermagem da USP, São Paulo, v. 34, n. 1, p. 16-25, 2000.

MORSE J. M. Preventing patient falls. Thousand Oaks: Sage, 1997.

NASCIMENTO, C. C. P. et al. Indicadores de resultados da assistência: análise dos eventos adversos durante a internação hospitalar. Revista Latino-Americana de Enfermagem, Ribeirão Preto, v. 16, n. 4, p. 746-751, 2008.
PHILLIPS, L. D. Controle de infecção: manual de terapia intravenosa. 2. ed. Porto Alegre: Artmed, 2001. p. 140-166.

POTTER, P. A.; PERRY, A. G. Fundamentos de enfermagem. 5. ed. Rio de Janeiro: Elsevier, 2009.

SETZ, V. G.; D'INNOCENZO, M. Avaliação da qualidade dos registros de enfermagem no prontuário por meio da auditoria. Acta Paulista de Enfermagem, São Paulo, v. 22, n. 3, p. 313-317, 2009.

TRNOBRANSKI, P. H. Nurse-patient negotiation: assumption or reality? Journal of Advanced Nursing, London, v. 19, n. 4. p. 733-737, 1993.

VITURI, D. W. Desenvolvimento e validação de um instrumento para avaliação da qualidade do cuidado de enfermagem. 2007. 235 f. Dissertação (Mestrado) - Universidade Estadual de Maringá, Maringá, 2007. 